\title{
Review
}

\section{Management of locally advanced prostate cancer}

\author{
Heather Payne \\ Department of Oncology, UCLH NHS Foundation Trust, London NW1 2PG, UK
}

\begin{abstract}
The management of all stages of prostate cancer is an increasingly complex process and involves a variety of available treatments and many disciplines. Despite prostate-specific antigen (PSA) testing, the presentation of prostate cancer at a locally advanced stage is common in the UK, accounting for one-third of all new cases. There is no universally accepted definition of locally advanced prostate cancer; the term is loosely used to encompass a spectrum of disease profiles that show high-risk features. Men with high-risk prostate cancer generally have a significant risk of disease progression and cancer-related death if left untreated. High-risk patients, including those with locally advanced disease, present two specific challenges. There is a need for local control as well as a need to treat any microscopic metastases likely to be present but undetectable until disease progression. The optimal treatment approach will therefore often necessitate multiple modalities. The exact combinations, timing and intensity of treatment continue to be strongly debated. Management decisions should be made after all treatments have been discussed by a multidisciplinary team (including urologists, oncologists, radiologists, pathologists and nurse specialists) and after the balance of benefits and side effects of each therapy modality has been considered by the patient with regard to his own individual circumstances. This article reviews the current therapy options.
\end{abstract}

Asian Journal of Andrology (2009) 11: 81-87. doi: 10.1038/aja.2008.9; published online 1 December 2008.

Keywords: antiandrogens, prostate cancer, prostatectomy

\section{Introduction}

The management of all stages of prostate cancer is an increasingly complex process. It requires a variety of available treatments and the involvement of many disciplines. Despite prostate-specific antigen (PSA) testing, the presentation of prostate cancer at a locally advanced stage is common in the UK, accounting for onethird of all new cases.

To date, there is no universally accepted definition of locally advanced prostate cancer. The term, however, is loosely used to encompass a spectrum of disease profiles that may include any of the following:

Correspondence to: Dr Heather Payne, Department of Oncology, UCLH NHS Foundation Trust, 250 Euston Road, London NW1 2PG, UK.

Fax: +44-20-7224-5706

E-mail: heather_payne@blueyonder.co.uk

Received: 17 September 2008 Accepted: 19 September 2008

Published online: 1 December 2008
(A) Clinical stage American Joint Committee on Cancer (AJCC) T3 (tumours extending to the periprostatic area or into seminal vesicles), T4 (larger tumours invading the external sphincter, bladder neck, rectum, levator ani muscles or fixed to the pelvic side wall) or N1 (regional pelvic lymph node involvement associated with any local T stage), without evidence of distant metastases M0 [1].

(B) Clinical stages T1 and T2 ('localized') at diagnosis, where so-called 'high-risk' features indicate the likelihood of extraprostatic invasion or clinically undetectable metastatic disease.

(C) Pathological stage pT2 or pT3 disease, with highrisk features owing to upstaging from additional pathological information after radical prostatectomy.

The degree of uncertainty regarding the definition of locally advanced prostate cancer was recently shown in a survey of more than 150 oncologists and urologists in the UK in which respondents were asked to define the term [2]. A total of 95 different answers were given, including various combinations of pretreatment PSA, 
Gleason score (GS), tumour-node-metastasis staging and other pathological features. The inconsistencies between physicians' responses indicate the need for a clearer definition of locally advanced prostate cancer. They also suggest that the current thinking underlying disease management is based primarily on a combination of patient risk factors. Despite their limitations, clinical stage, serum PSA and Gleason sum score form the principal basis for therapeutic recommendations. A categorization that defines three risk groups (high, intermediate and low risk) according to established prognostic factors has been described by D'Amico et al. [3]. High-risk patients were defined as men with a greater than $50 \%$ chance of failure after primary therapy, including any patient with AJCC stage T3a or above, pretreatment PSA levels higher than $20 \mathrm{ng} \mathrm{mL} \mathrm{m}^{-1}$ or a biopsy $\mathrm{GS} \geq 8$. The group of high-risk patients in this definition more accurately reflects the options and challenges of treating 'locally advanced' prostate cancer. This group, rather than the anatomical stage, is the focus of this article.

There are currently a number of possible management options for high-risk prostate cancer. Some patients are offered radical radiotherapy, radical prostatectomy or androgen deprivation therapy, either alone or in combination with local treatment. For other patients, a period of watchful waiting, enrolment in a clinical trial or intervention with a novel or experimental therapeutic modality may be recommended. These therapies can be given with either palliative or radical intent. Men with high-risk prostate cancer generally have a significant risk of disease progression, which may result in cancerrelated death if left untreated. High-risk patients, including those with locally advanced disease, present two specific challenges. There is a need for local control, as well as a need to treat any microscopic metastases likely to be present but undetectable until disease progression. The optimal treatment approach will therefore often necessitate multiple modalities. The exact combinations, timing and intensity of treatment continue to be strongly debated. Management decisions should be made after all treatments have been discussed by a multidisciplinary team (including urologists, oncologists, radiologists, pathologists and nurse specialists) and the balance of benefits and side effects of each therapy modality has been considered by the patient with regard to his own circumstances.

\section{Watchful waiting/androgen deprivation therapy}

Watchful waiting involves observation with late palliative treatment (usually hormone therapy) for men who develop symptoms of progressive disease. There are two main methods of achieving prostate cancer control using hormone therapy. The first is to remove the supply of endogenous testosterone with castration-based therapy. This can be achieved with either bilateral surgical orchidectomy or a medical approach using luteinizing hormone-releasing hormone agonists (LHRHa). These drugs act by suppressing testosterone production from the testes through positive feedback of LHRH at the pituitary level. They are usually administered either monthly or tri-monthly by depot injections. There may be an initial testosterone flare at the time of administration of the first implant; this can be prevented by treatment with an antiandrogen drug (see below) to block testosterone at the cellular level for a few weeks before and after the first injection. The side effects of LHRHa include erectile dysfunction, loss of libido, hot flushes, osteopaenia/ osteoporosis, weight gain and breast swelling.

An alternative approach is to reduce the effect of endogenous hormones with drugs that block androgen receptors (antiandrogens). The antiandrogens (e.g., $150 \mathrm{mg}$ bicalutamide) are given orally and may have advantages with regard to toxicity over castration-based therapy for some men. Bicalutamide $150 \mathrm{mg}$ helped maintain physical capacity and bone mineral density; however, gynaecomastia and mastalgia are frequently associated toxicities.

Both treatments have proven effective for locally advanced prostate cancer, and the different side effect profiles can allow clinicians and patients to choose the best approach to maintain quality of life for each patient.

There has been some debate as to whether there are advantages of immediate androgen deprivation therapy as opposed to an initial period of observation or watchful waiting. Some early nonrandomized studies concluded that immediate orchidectomy was not associated with a survival advantage compared with therapy delayed until metastatic progression [4, 5]. However, analysis of a randomized controlled trial involving 943 men not suitable for curative treatment by the Medical Research Council suggested benefits for immediate vs delayed hormonal treatment [6]. The rates of distant progression were $26 \%$ for men treated with immediate castration-based therapy and $46 \%$ for men whose treatment was deferred. This study showed an apparent advantage in treating patients immediately with androgen deprivation therapy in terms of distant progression, but mortality was significantly changed only in the subgroup with M0 (locally advanced) disease. In another study conducted by the European Organisation for Research and Treatment of Cancer (EORTC) [7], 985 men with newly diagnosed prostate cancer (T0-T4, N0-N2, M0) were randomly assigned to immediate vs delayed hormone therapy given at the time of symptomatic progression. The group that received immediate androgen deprivation showed a modest but statistically significant increase in overall survival, but no significant difference was seen in prostate cancer mortality or symptom-free survival. The study concluded that any 
advantages in survival should be weighed on an individual basis against the side effects associated with long-term androgen deprivation.

The nonsteroidal antiandrogen, bicalutamide, was also investigated in this setting as part of a multicentre, international Early Prostate Cancer (EPC) study [8]. This trial was designed to evaluate the efficacy and tolerability of adding $150 \mathrm{mg}$ bicalutamide once daily to standard care (prostatectomy, radiotherapy or watchful waiting) in 8113 patients with localized or locally advanced nonmetastatic prostate cancer. The primary end points were objective progression-free survival (PFS) and overall survival. The median follow-up time at the most recent analysis was 7.4 years. Exploratory analyses were conducted to determine the efficacy of bicalutamide in clinically relevant subgroups. There was a trend towards increased survival for the subgroup with locally advanced disease treated with $150 \mathrm{mg}$ bicalutamide, who would otherwise have undergone watchful waiting (hazard rate [HR] 0.81; 95\% confidence interval $[\mathrm{CI}] 0.66-1.01 ; P=0.06)$. A significant improvement in objective PFS in favour of 150 $\mathrm{mg}$ bicalutamide was also shown for this watchful-waiting group (HR 0.60; 95\% CI 0.49-0.73; $P<0.0001$ ).

The majority of men with locally advanced prostate cancer face a risk of significant disease progression that can cause debilitating symptoms, including bone pain, fracture, spinal cord compression and urinary dysfunction. This is especially true for men who present with other unfavourable disease characteristics associated with more rapid progression, such as a GS $\geq 8$ or a PSA above 20 . Although this must be weighed against the toxicities of hormone therapy, the advantages of early therapy usually outweigh the side effects for men with high-risk disease.

\section{Radical prostatectomy}

Radical prostatectomy has traditionally been reserved for cases with a low risk of significant extraprostatic spread and lymph node involvement and therefore has not been a widely accepted treatment for men with clinical locally advanced prostate cancer. A publication from the Mayo Clinic [9] challenges this view with results of a large group of men with clinical stage T3 disease who were treated with primary radical prostatectomy. In this series, $25 \%$ of the men had pathologically organ-confined tumours likely to be cured by surgical therapy alone. Cancer-specific survival rates at 5, 10 and 15 years were $93 \%, 84 \%$ and $74 \%$, respectively. The authors concluded that excellent long-term survival was possible with radical prostatectomy and additional adjuvant therapy for some men with locally advanced prostate cancer.

The definition of high-risk prostate cancer can be extended to include men who present with clinically localized disease but are found to have pathological extraprostatic disease (pT3), positive surgical margins, lymph node involvement or high-grade disease after radical prostatectomy. How and when to treat men with these adverse features after radical prostatectomy is one of the most challenging questions. There is a known risk of tumour recurrence, both at local and at distant sites. The combination of adjuvant radiotherapy and/or systemic therapies and the optimal timing and duration of these treatments remain unclear despite having the results of two randomized studies. The EORTC 22911 by Bolla et al. [10] was designed to investigate the benefit of immediate postoperative radiotherapy in patients with $\mathrm{pT} 3$ disease or positive surgical margins as opposed to a 'wait and see' policy with salvage radiotherapy offered in the event of local recurrence. A total of 502 patients were randomized to the immediate treatment arm and received a dose of 60 Gy with nonconformal radiotherapy. Of the 503 men randomized to the delayed radiotherapy arm, 113 received salvage radiation treatment. At a median follow-up of 5 years, a significant advantage was reported for adjuvant treatment in terms of biochemical PFS (72\% vs. 51\%; HR 0.52; 95\% CI: $0.42-0.64 ; P<0.0001)$ and clinical PFS (83\% vs. 75\%; HR 0.68; 95\% CI: 0.52-0.89; $P=0.004$ ). There was no difference in overall survival (in the region of $92 \%$ for both groups at 5 years). In terms of toxicity, the cumulative event incidence of grade three toxicity at 5 years was $2.6 \%$ in the wait-and-see group and $4.2 \%$ in the immediate radiotherapy group $(P=0.0726)$. SWOG 8794 [11] also reported the results of 425 men with pT3 disease who were randomized for adjuvant radiotherapy to the prostate bed (60-64 Gy) or observation and subsequent salvage therapy. The authors also reported a significant improvement in biochemical control (HR 0.43 [0.31-0.58]; $P<0.001$ ) for the group treated with immediate adjuvant radiotherapy. This study had a longer median follow-up (10.6 years), and adjuvant radiotherapy was associated with a trend towards better metastasis-free survival (HR 0.75 $[0.55-1.02] ; P=0.06$ ) and overall survival (HR $0.80[0.58$ $1.09] ; P=0.16)$. Proctitis $(3.3 \%$ vs. $0 \%)$, urethral stricture $(17.8 \%$ vs. $9.5 \%)$ and urinary incontinence $(6.5 \%$ vs. $2.8 \%)$ were seen more often in the adjuvant radiotherapy arm. These studies provide evidence that postoperative radiotherapy can reduce the risk of PSA and clinical failure, and the SWOG study suggested a trend towards an increase in overall survival for men treated with adjuvant radiation. However, there have been changes in clinical practice since these studies were designed, and it is now typical to offer salvage radiotherapy for PSA rather than clinical relapse. This 'early' treatment approach could lead to an improvement in the efficacy of salvage therapy and enable better selection for men who would benefit the most from additional treatment. The dose of radiotherapy has also 
typically increased to 64-66 Gy and is usually delivered with a more precise three-dimensional conformal (3DCRT) or the intensity modulated radiotherapy (IMRT) approach, which may reduce some associated morbidity by sparing the surrounding normal structures. These current standards of care are being investigated in the ongoing Radiotherapy and Androgen Deprivation In Combination After Local Surgery (RADICALS) [12] study, which examines the timing of postoperative radiotherapy with a comparison between adjuvant radiotherapy and observation with early salvage radiation for biochemical failure. RADICALS added a further randomization to investigate the addition and duration of concomitant hormone therapy, which remains another unanswered question in the postoperative setting. Although there are many studies that have shown benefits in men receiving primary radiotherapy to the prostate, little data are available on the role of hormone therapy in men receiving postoperative radiation. The results from another study, the Radiation Therapy Oncology Group (RTOG) 96-01, are eagerly awaited and may help to answer this important question. This study includes 840 men with PSA failure after radical prostatectomy. They were randomized between early salvage radiotherapy alone and early salvage radiotherapy plus 2 years of $150 \mathrm{mg}$ daily bicalutamide.

The results of treatment with hormone therapy alone for men with high-risk disease after prostatectomy are uncertain. One small but highly significant study by Messing et al.[13] showed benefits for men with pathological lymph node involvement.

The ECOG 7887 [14] trial compared adjuvant hormone ablation after radical prostatectomy and deferred hormonal therapy in patients with nodal metastases. A total of 98 patients with locally advanced prostate cancer (T1-T2/N+ disease) who had undergone pelvic lymphadenectomy were included in the study. These patients were randomized to receive adjuvant hormone ablation $(n=47)$ or to be followed until disease progression $(n=51)$ and then given hormonal therapy. At a median follow-up of 11.9 years, adjuvant hormone ablation increased the survival by 2.6 years compared with surgery alone in node-positive patients. The median survival in the adjuvant hormone ablation and deferred treatment groups was 13.9 and 11.3 years, respectively. The men assigned immediate hormone therapy had a significant improvement in overall survival (HR 1.84; 95\% CI 1.01-3.35, $P=0.04$ ), prostate-cancerspecific survival (4.09 [1.76-9.49], $P=0.0004)$, and progression-free survival (3.42 [1.96-5.98], $P<0.0001$ ).

Treatment may therefore be best achieved with a stratified approach, because risks for local and distant recurrence will vary depending on other prognostic factors. As a consequence, the benefits of radiotherapy and hormone therapy will differ. Ongoing studies will help to define optimal management for men after radical prostatectomy with the aim of avoiding the morbidity of over-treatment and the risks of disease progression with under-treatment.

\section{Radiotherapy}

External beam radiotherapy is a well-established treatment for all stages of prostate cancer. Some men with a high risk of locally advanced disease may experience early disease progression with this treatment modality alone [14]. GS, T stage and pathologic lymph node status have been described as major independent predictors of death due to prostate cancer in men treated with external beam radiotherapy. Roach et al. [15] used these three prognostic factors to analyse the outcomes of 1557 men who received radiotherapy alone in the RTOG clinical trials between 1975 and 1992. They identified four prognostic subgroups:

Risk group 1: GS $=2-6, \mathrm{~T} 1-2 / \mathrm{N} \times$

Risk group 2: $\mathrm{GS}=2-6, \mathrm{~T} 3 / \mathrm{N} \times$; or $\mathrm{GS}=2-6, \mathrm{~N}+$, or $\mathrm{GS}=7, \mathrm{~T} 1-2 / \mathrm{N} \times$

Risk group 3: $\mathrm{T} 3 \mathrm{~N} \times, \mathrm{GS}=7$; or $\mathrm{N}+, \mathrm{GS}=7$, or $\mathrm{T} 1-$ $2 / \mathrm{N} \times, \mathrm{GS}=8-10$

Risk group 4: $\mathrm{T} 3 \mathrm{~N} \times, \mathrm{GS}=8-10$, or $\mathrm{N}+, \mathrm{GS}=8-10$

The 5-, 10- and 15-year disease-specific survival was $96 \%, 86 \%$ and $72 \%$ for group $1 ; 94 \%, 75 \%$ and $61 \%$ for group 2; $83 \%, 62 \%$ and $39 \%$ for group 3; and $64 \%, 34 \%$ and $27 \%$ for group 4 .

Zagars et al. [16] also showed that the risk of progression or relapse after radical radiotherapy increases with poor prognostic factors such as an initial PSA level $\geq 10$ and any single GS $\geq 4$ on the biopsy. These studies show that conventional radiotherapy alone is often inadequate as treatment for high-risk prostate cancer.

There is evidence that an increase in radiation dose is associated with increased cancer cell death in prostate cancer. The traditional two-dimensional technique of treatment planning and delivery, however, is limited by the normal tissue toxicity of the surrounding structures, namely the bladder, rectum and bowel. New technological advances such as CT-planned 3DCRT have improved the precision of external beam radiotherapy (EBRT) by more accurately shaping the beams to the target volume whilst reducing radiation to the dose-limiting structures. The advantages of this technique were shown in a phase 3 randomized study comparing 3DCRT with conventional unshaped radiotherapy at a then-standard dose of 64 Gy [17]. A significant reduction in rectal toxicity was shown, without any alteration in treatment efficacy. This encouraged the investigation of higher doses of radiation in attempts to establish the optimal dose that could be safely delivered. A randomized study at the MD Anderson Cancer Center [18] 
comparing doses of 70 Gy and 78 Gy in 305 men with T1-T3 prostate cancer showed a significant improvement in 'freedom from failure', including biochemical failure in the group of men presenting with a PSA $>10$. In this higher-risk group, the freedom from failure rate was $62 \%$ for the 78 -Gy arm vs. $43 \%$ for men who received 70 Gy $(P=0.01)$. The rate of freedom from distant metastasis was higher for men with PSA levels $>10$ who were treated with a dose of 78 Gy $(98 \% v s .88 \%$ at 6 years, $P=0.056$ ). This study has therefore shown benefits from dose escalation for men with higher-risk tumours known to have a poorer outcome with traditional doses of radiotherapy. This observation has been supported by findings from the MRC RT01 study [19]. In this 3DCRT trial, 843 men were randomized to a standard dose of 64 Gy compared with an escalated dose of 74 Gy. Patients receiving the conventional dose had 5-year biochemical PFS rates of $60 \%$ compared with $71 \%$ in the dose-escalated arm. Advantages were also seen in terms of PFS and the decreased use of future androgen suppression. More recently, a further development has been reported using IMRT, which is an advanced form of 3DCRT that uses sophisticated computer-assisted technology to modify and shape the intensity of multiple radiotherapy beams during treatment to deliver very precise coverage of the target area whilst further sparing the surrounding sensitive tissues. This has allowed the use of doses $>80$ Gy in prostate cancer treatment. In another study of dose escalation, Zelefsky et al. [20] have shown that increasing the dose delivered to $>70$ Gy in men with intermediate and high-risk disease improved the 5-year actuarial PSA relapse-free survival rates from $50 \%$ to $70 \%$ and from $21 \%$ to $47 \%$, respectively.

Further developments in radiotherapy for prostate cancer have included studies investigating different fractionation schedules. There is increasing evidence that hypofractionated schedules, involving a smaller number of larger doses per fraction of radiation, could be more effective for treating prostate cancer. This reflects the recent finding that the radiobiological alpha/beta ratio for prostate cancer is low [21]. Conventionally, radiobiology states that the alpha/beta ratios for tumours are higher than those for the surrounding normal late-reacting tissue. Tissues with a lower alpha/beta ratio will undergo greater cell death by larger doses per fraction of radiotherapy than tissues with a higher ratio. This type of regime is the subject of ongoing clinical studies.

Another method of dose escalation involves highdose-rate (HDR) brachytherapy boost in combination with external beam irradiation. This involves the placement of hollow needles into the prostate, periprostatic tissues and seminal vesicles under ultrasound guidance. Dose conformity is enhanced by the generation of a steep dose gradient between the prostate and normal tissues and by controlling the length of time that a radioactive iridium192 source temporarily dwells at variable positions within the needles. HDR brachytherapy is commonly used in other tumour sites and allows large doses of radiation to be delivered within a few minutes. This technique allows the administration of a truly conformal and optimized dose that also takes organ movement into account. The results of HDR boost in combination with external beam radiotherapy are encouraging for high-risk patients. Martinez et al. [22] reported the pooled results from 1260 men with intermediate to high-risk prostate cancer treated with HDR boost (dose escalated from 5.5 Gy $\times 3$ to 15 Gy $\times 2)$ and EBRT (36-50 Gy + 1.8-2 Gy daily). At a median follow-up of 4.4 years, the 8 -year biochemical no evidence of disease status was $81 \%$.

These studies represent a wide variety of different methods of radiotherapy dose delivery and clearly show the advantages of dose escalation, especially for men with high-risk tumours. The optimal delivery of therapy, dose and fractionation schedules, however, has yet to be determined and is the subject of multiple ongoing clinical studies.

\section{Radiotherapy in combination with hormone treatment}

A further challenge in treating high-risk prostate cancer is the concurrent treatment of potential microscopic metastases at distant sites. This means that, despite improved local treatment, many men will ultimately progress to metastatic disease that can cause debilitating morbidity and increase the mortality. There is considerable evidence that the addition of systemic treatment in the form of hormone therapy with androgen suppression is superior to radiotherapy alone in patients with high-risk disease. The use of combination therapy has been shown to improve survival and increase the time to progression.

Neoadjuvant hormone therapy (NHT) prior to definitive radiotherapy is commonly used. The aims of NHT are to reduce the tumour bulk and potentially treat microscopic metastases together with the primary tumour. This can cause an average of $25 \%-30 \%$ cytoreduction of the prostate $[23,24]$ and potentially allow smaller fields of radiotherapy to be used while sparing the surrounding normal tissues. There have also been reports that there may be a sensitizing effect between hormone therapy and radiation treatment. There are several theories as to the mechanism of this effect, including the hypothesis that reduction of tumour bulk improves oxygenation and therefore increases radiation sensitivity and movement within the cell cycle, thereby increasing apoptosis $[25,26]$. There is also clinical evidence to support this treatment approach. The RTOG 
86-10 [27] study investigated the addition of hormone therapy (goserelin and flutamide) for 2 months before and 2 months during radiotherapy compared with radiation treatment alone in 456 men with locally advanced prostate cancer [27]. At a median follow-up of 6.7 years, the patients in the combined modality arm had a significantly improved 5-year cause-specific survival of $90 \%$ vs. $85 \%$. A subgroup analysis showed that men with Gleason sum 6 tumours had an overall survival advantage at 5 years of $70 \%$ vs. $52 \%$.

Adjuvant androgen suppression immediately after radical radiotherapy has been shown to significantly increase overall survival and PFS and significantly reduce local progression, distant metastases and biochemical progression in several large, randomized studies using goserelin. The EORTC 22863 trial evaluated the effectiveness of adjuvant therapy with $3.6 \mathrm{mg}$ goserelin initiated at the onset of radiotherapy and continued for 3 years in patients with high-risk nonmetastatic prostate cancer [28]. A total of 415 patients were randomized to receive either radiotherapy with immediate hormone treatment or radiotherapy alone with hormonal treatment for disease progression. Results reported after a median follow-up of 5.5 years showed a significant improvement in overall survival ( $78 \%$ vs. $62 \%)$ and disease-free survival $(74 \%$ vs. $40 \%)$ in favour of immediate adjuvant treatment. Further data are available from other studies, including the RTOG 85-10 [29], in which 977 men were randomly chosen to receive either pelvic radiation plus 3.6 $\mathrm{mg}$ goserelin (started during the last week of radiotherapy to be continued indefinitely every month) or radiotherapy alone with hormonal treatment commencing at relapse. Results at a median follow-up of 7.6 years indicated that adjuvant goserelin significantly improved the absolute survival compared with radiation monotherapy (estimated 10 -year survival rate $49 \%$ vs. $39 \% ; P=0.002$ ). The greatest benefits were seen in the subgroups with high Gleason grades (grades 8-10). The results of this highly significant study show important overall survival benefits. Furthermore, the results from these studies have changed the clinical practice for locally advanced prostate cancer to include a combined modality approach. There are uncertainties regarding the optimal timing and duration of hormone therapy. Timing has varied among different trials. Goserelin was added during the final week of RTOG 85-31 [29] and during the first week of EORTC 22863 [28]. There were also differences in the duration of adjuvant goserelin therapy in these studies. The quality of life of the patient is an important factor when deciding on the duration of therapy, and any long-term side effects must also be considered. We await further results of these and other studies to determine the optimal duration of hormone treatment. The introduction of new radiotherapy techniques, as described above, has allowed further dose escalation. Again, this combination may lead to even greater improvement in the outcome.

Data from the third analysis of the EPC study indicated that $150 \mathrm{mg}$ bicalutamide adjuvant treatment to radiotherapy has resulted in significantly improved overall survival compared with radiotherapy alone (HR, 0.65; $P=0.03$ ) for men with locally advanced prostate cancer at a median follow-up of 7.4 years [30]. The prostate cancer mortality for this subgroup was $24 \%$ for patients treated with radiotherapy alone compared with $16 \%$ for men treated with combined modality therapy. This represents a significant overall survival benefit for non-castrationbased hormonal therapy given as adjuvant treatment to radiotherapy. These results give clinicians and patients a choice regarding which adjuvant hormone therapy to use without the concern of reducing treatment efficacy.

\section{Conclusion}

The management of locally advanced prostate cancer remains a challenge for urologists and oncologists. There is a growing body of evidence suggesting that combination modality treatment may improve disease-specific outcomes and the overall survival rate over single modalities for select patient groups. The evidence base to determine optimal therapies and their timing is rapidly growing, and we eagerly await the results of the trials of conventional combinations as well as the newer targeted drugs and chemotherapy.

\section{References}

1 American Joint Committee on Cancer. Prostate. AJCC Cancer Staging Manual. New York, NY: Springer; 2002. p309-16.

2 Payne H, Gillatt D. Differences and commonalities in the management of locally advanced prostate cancer: results from a survey of oncologists and urologists in the UK. BJU Int 2007; 3: 545-53.

3 D'Amico AV, Whittington R, Malkowicz SB, Schultz D, Blank K, et al. Biochemical outcome after radical prostatectomy, external beam radiation therapy, or interstitial radiation therapy for clinically localized prostate cancer. JAMA 1998; 280: 969-97.

4 Rana A, Chisholm GD, Khan M, Rashwan HM, Elton RA. Conservative management with symptomatic treatment and delayed hormonal manipulation is justified in men with locally advanced carcinoma of the prostate. Br J Urol 1994; 74: 637-41.

5 Parker MC, Cook A, Riddle PR, Fryatt I, O'Sullivan J, et al. Is delayed treatment justified in carcinoma of the prostate? Br J Urol 1985; 57: 724-8.

6 Medical Research Council Prostate Cancer Working Party Investigators Group. Immediate versus deferred treatment for advanced prostatic cancer: initial results of the Medical Research Council Trial. The Medical Research Council Prostate Cancer Working Party Investigators Group. Br J Urol 1997; 79:235-46.

7 Studer UE, Whelan P, Albrecht W, Casselman J, de Reijke T, et al. Immediate or deferred androgen deprivation for patients with 
prostate cancer not suitable for local treatment with curative intent: European Organisation for Research and Treatment of Cancer (EORTC) Trial 30891. J Clin Oncol 2006; 24: 1868-76.

8 McLeod DG, Iversen P, See WA, Morris T, Armstrong J, et al; Casodex Early Prostate Cancer Trialists' Group. Bicalutamide $150 \mathrm{mg}$ plus standard care vs standard care alone for early prostate cancer. BJU Int 2006; 97: 247-54.

9 Amling CL, Leibovich BC, Lerner SE, Bergstralh EJ, Blute ML, et al. Primary surgical therapy for clinical stage T3 adenocarcinoma of the prostate. Semin Urol Oncol 1997; 15: 215-21.

10 Bolla M, van Poppel H, Collette L, van Cangh P, Vekemans K, et al.; European Organization for Research and Treatment of Cancer. Postoperative radiotherapy after radical prostatectomy: a randomised controlled trial (EORTC trial 22911). Lancet 2005; 366: 572-8.

11 Thompson IM Jr, Tangen CM, Paradelo J, Lucia MS, Miller G, et al. Adjuvant radiotherapy for pathologically advanced prostate cancer: a randomized clinical trial. JAMA 2006; 296: 2329-35.

12 Parker C, Sydes MR, Catton C, Kynaston H, Logue J, et al; (RADICALS Trial Management Group). Radiotherapy and androgen deprivation in combination after local surgery (RADICALS): A new Medical Research Council/National Cancer Institute of Canada phase III trial of adjuvant treatment after radical prostatectomy. BJU Int 2007; 99: 1376-9.

13 Messing EM, Manola J, Yao J, Kiernan M, Crawford D, et al; Eastern Cooperative Oncology Group study EST 3886. Immediate versus deferred androgen deprivation treatment in patients with node-positive prostate cancer after radical prostatectomy and pelvic lymphadenectomy. Lancet Oncol 2006; 7: 472-9.

14 Shipley WU, Thames HD, Sandler HM, Hanks GE, Zietman $\mathrm{AL}$, et al. Radiation therapy for clinically localized prostate cancer: a multi- institutional pooled analysis. JAMA 1999; 281: 1598-604.

15 Roach M, Lu J, Pilepich MV, Asbell SO, Mohiuddin M, et al. Four prognostic groups predict long-term survival from prostate cancer following radiotherapy alone on Radiation Therapy Oncology Group clinical trials. Int J Radiat Oncol Biol Phys 2000; 47: 609-15.

16 Zagars GK, Pollack A, Von Eschenbach AC. Management of unfavorable locoregional prostate carcinoma with radiation and androgen ablation. Cancer 1997; 80: 764-75.

17 Dearnaley DP, Khoo VS, Norman AR, Meyer L, Nahum A, et al. Comparison of radiation side-effects of conformal and conventional radiotherapy in prostate cancer: a randomised trial. Lancet 1999; 353: 267-72.

18 Pollack A, Zagars GK, Starkschall G, Antolak JA, Lee JJ, et al. Prostate cancer radiation dose response: results of the M. D. Anderson phase III randomized trial. Int J Radiat Oncol Biol
Phys 2002; 53: 1097-105.

19 Dearnaley DP, Sydes MR, Graham JD, Aird EG, Bottomley D, et al.; RT01 collaborators. Escalated-dose versus standard-dose conformal radiotherapy in prostate cancer: first results from the MRC RT01 randomised controlled trial. Lancet Oncol 2007; 8: 475-87.

20 Zelefsky MJ, Fuks Z, Hunt M, Lee HJ, Lombardi D, et al. High dose radiation delivered by intensity modulated conformal radiotherapy improves the outcome of localized prostate cancer. J Urol 2001; 166: 876-81.

21 Bentzen SM, Ritter MA. The alpha/beta ratio for prostate cancer: what is it, really? Radiother Oncol 2005; 76: 1-3.

22 Martinez AA, Demanes DJ, Galalae R, Vargas C, Bertermann $\mathrm{H}$, et al. Lack of benefit from a short course of androgen deprivation for unfavorable prostate cancer patients treated with an accelerated hypofractionated regime. Int J Radiat Oncol Biol Phys 2005; 62: 1322-31.

23 Henderson A, Langley SE, Laing RW. Is bicalutamide equivalent to goserelin for prostate volume reduction before radiation therapy? A prospective, observational study. Clin Oncol (R Coll Radiol) 2003; 15: 318-21.

24 Zelefsky MJ, Leibel SA, Burman CM, Kutcher GJ, Harrison A, et al. Neoadjuvant hormonal therapy improves the therapeutic ratio in patients with bulky prostatic cancer treated with threedimensional conformal radiation therapy. Int J Radiat Oncol Biol Phys 1994; 29: 755-61.

25 Linberg R, Conover CD, Shum KL, Shorr RG. Increased tissue oxygenation and enhanced radiation sensitivity of solid tumors in rodents following polyethylene glycol conjugated bovine hemoglobin administration. In Vivo 1998; 12: 167-73.

26 Hara I, Miyake H, Yamada Y, Takechi Y, Hara S, et al. Neoadjuvant androgen withdrawal prior to external radiotherapy for locally advanced adenocarcinoma of the prostate. Int J Urol 2002; 9: 322-8.

27 Pilepich MV, Winter K, John MJ, Mesic JB, Sause W, et al. Phase III radiation therapy oncology group (RTOG) trial 86-10 of androgen deprivation adjuvant to definitive radiotherapy in locally advanced carcinoma of the prostate. Int J Radiat Oncol Biol Phys 2001; 50: 1243-52.

28 Bolla M, Collette L, Blank L, Warde P, Dubois JB, et al. Long-term results with immediate androgen suppression and external irradiation in patients with locally advanced prostate cancer (an EORTC study): a phase III randomised trial. Lancet 2002; 360: 103-6.

29 Pilepich MV, Winter K, Lawton CA, Krisch RE, Wolkov HB, et al. Androgen suppression adjuvant to definitive radiotherapy in prostate carcinoma-long-term results of phase III RTOG 85-31. Int J Radiat Oncol Biol Phys 2005; 61: 1285-90.

30 McLeod DG, Iversen P, See WA, Morris T, Armstrong J, et al. Bicalutamide $150 \mathrm{mg}$ plus standard care vs standard care alone for early prostate cancer. BJU Int 2006; 97: 247-54. 